Article

\title{
Diet Quality and Nutrient Intake of Urban Overweight and Obese Primarily African American Older Adults with Osteoarthritis
}

\author{
Sevasti Vergis ${ }^{1,2,3}$, Linda Schiffer ${ }^{4}$, Tiffany White ${ }^{2}$, Andrew McLeod ${ }^{2,4}$, Neda Khudeira ${ }^{1,2}$, \\ Andrew Demott 5 (D), Marian Fitzgibbon ${ }^{4,6,7}$, Susan Hughes ${ }^{5,8}$ and Lisa Tussing-Humphreys 1,7,* \\ 1 Department of Medicine, 1747 W. Roosevelt Road, Chicago, IL 60608, USA; svergi3@uic.edu (S.V.); \\ nedakhudeira@gmail.com (N.K.) \\ 2 Department of Kinesiology and Nutrition, University of Illinois at Chicago, 1919 W. Taylor Street, \\ Chicago, IL 60612, USA, white35@uic.edu (T.W.); amcleo2@uic.edu (A.M.) \\ 3 Honors College, University of Illinois at Chicago, 828 S. Halsted Street, Chicago, IL 60607, USA \\ 4 Institute for Health Research and Policy, 1747 W. Roosevelt Road, Chicago, IL 60608, USA; \\ lschiff@uic.edu (L.S.); mlf@uic.edu (M.F.) \\ 5 Center for Research on Health and Aging, Institute for Health Research and Policy, University of Illinois at \\ Chicago, 1747 W. Roosevelt Road, Chicago, IL 60608, USA; ademot1@uic.edu (A.D.); shughes@uic.edu (S.H.) \\ 6 Department of Pediatrics, University of Illinois at Chicago, 1853 W. Polk Street, Chicago, IL 60612, USA \\ 7 University of Illinois Cancer Center, University of Illinois at Chicago, 818 S. Wolcott Avenue, \\ Chicago, IL 60612, USA \\ 8 Department of Community Health Sciences, School of Public Health, University of Illinois at Chicago, \\ 1603 W. Taylor St, Chicago, IL 60612, USA \\ * Correspondence: ltussing@uic.edu; Tel.: +1-312-355-5521
}

Received: 30 January 2018; Accepted: 11 April 2018; Published: 13 April 2018

\begin{abstract}
Diet quality may be a unique target for preventing and managing obesity-related osteoarthritis (OA). Using the Healthy Eating Index-2010 (HEI-2010), this study examined the nutrient intake and diet quality of 400 urban overweight and obese primarily African American older adults with self-reported lower extremity OA. Associations between sociodemographic and health-related factors and diet quality were explored. Participants (mean age 67.8 years, SD 5.9) were included. Habitual dietary intake was assessed using a food frequency questionnaire (FFQ). Nutrient intake and diet quality were calculated from the FFQ. Results indicated that diet quality needs improvement (HEI-2010: 66.3 (SD 10.5)). Age, body mass index, employment (multivariable model only), and OA severity (bivariate model only) were significant predictors of HEI-2010 total score in linear models. Mean intakes for fiber, calcium, and vitamin D were below recommendations, while percentage of calories as total fat exceeded recommendations. These findings can inform future dietary intervention trials and public health messaging for a sub-population at a high risk for obesity-related OA.
\end{abstract}

Keywords: diet quality; nutrient intake; African Americans; older adults; osteoarthritis

\section{Introduction}

The population of older adults in the United States (U.S.), specifically adults over the age of 65 years, rose dramatically from 35 million in 2000 to 49.2 million in 2016 [1]. This shift in U.S. demographics comes with an increase in the incidence and prevalence of chronic diseases that place a significant toll on an individual's quality of life as they age as well as on the U.S. healthcare system [2]. Osteoarthritis (OA) is one such condition affecting over 30 million older adults in the U.S., with African Americans disproportionately burdened by large joint (i.e., kip, knee) OA $[3,4]$. This health inequity is largely attributed to higher rates of overweight and obesity among older African American adults [5], 
particularly African American women, given excess body weight is a significant modifiable risk factor for OA [6].

There are currently two major causal theories explaining the association between overweight/obesity and OA. The mechanical theory focuses on the effects of loading and cartilage degradation-specifically, the repetitive application of a higher load on large joints (i.e., knee and hip) that leads to the degeneration of articular cartilage and sclerosis of the subchondral bone [7]. The second theory, known as the metabolic theory, surmises that OA arises through an indirect action of elevated pro-inflammatory cytokines stemming from increased adipose tissue that leads to joint degradation or, along with the mechanical stress, exacerbates joint degeneration [8]. Thus, interventions aimed at reducing excess body weight and systemic inflammation may have a significant role in OA prevention, progression, and symptom management.

Diet is a common intervention target to reduce excess body weight and systemic inflammation, and has been the focus of several studies in older overweight and obese adults with OA. The majority of studies have focused on calorie restriction to promote weight loss, demonstrating promising short-term effects for weight loss, systemic inflammation, and physical function $[9,10]$. However, difficulties maintaining weight loss in the long term [11] and the untoward effects of calorie restriction on lean muscle mass [12] has led researchers and clinicians to seek alternative dietary approaches that may be more maintainable but comparable in regard to body fat and inflammatory results [13].

Diet quality is a relatively new concept in nutritional epidemiology focused on the quality and synergy of whole foods included in a diet pattern versus single nutrients [14]. Diet quality can be determined using data-driven approaches such as principal components analysis as well as a priori methods that assess adherence to existing population-based dietary recommendations (e.g., United States Department of Agriculture's (USDA) Dietary Guidelines for Americans (DGAs)). One common a priori method for determining diet quality is the Healthy Eating Index (HEI) [15]. HEI assesses adherence to a given set of DGAs; higher scores reflect greater adherence [16]. Operationalizing and quantifying diet quality allows one to examine associations with health status [14]. In recent studies, improving diet quality was associated with less weight gain over time [17], greater weight loss [18], and reduced systemic inflammation independent of weight loss [19]. Moreover, superior diet quality is associated with lower OA prevalence [20] and reduced risk for symptomatic knee OA in women [13]. Together, these findings suggest that improving diet quality may be a key target for preventing and managing obesity-related OA.

In order to design effective interventions that target diet quality, an understanding of current diet quality is essential. To date, no studies have examined diet quality in older overweight and obese African American adults with OA-a subpopulation at a disproportionately burdened by OA $[3,4]$. However, we do know from general population-based studies that older African American adults have lower diet quality based on HEI compared to other racial/ethnic groups [21]. The primary aim of this study was to describe the diet quality (HEI-2010) and nutrient intake of urban overweight and obese primarily African American older adults with self-reported lower extremity OA. The secondary aim was to examine associations between sociodemographic and health-related factors and diet quality. Such data will help to guide the development of tailored lifestyle interventions to promote healthy aging in populations at the greatest risk for OA [22].

\section{Materials and Methods}

\subsection{Study Design and Setting}

This study is a cross-sectional analysis of data collected at baseline from the Fit \& Strong! Plus comparative effectiveness trial (R01AG039374; NCT03180008) that was designed to compare the impact of physical activity versus physical activity plus dietary weight management on body weight, diet quality, physical activity, and OA symptoms in overweight and obese older adults with self-reported lower extremity OA. A detailed description of the trial's design and methods has been 
published elsewhere [23]. Briefly, subjects were recruited through advertisements and presentations at various community facilities in the city of Chicago including Chicago Park District sites and City of Chicago senior centers; emails were also sent from the Arthritis Foundation to listserv members residing in Chicago. The study was reviewed and approved by the University of Illinois at Chicago Institutional Review Board. All subjects provided written informed consent prior to study participation.

\subsection{Study Population and Eligibility Criteria}

Interested individuals were screened for eligibility over the phone. Eligibility criteria included: self-reported diagnosis of lower extremity OA described as pain in or around at least one knee or hip most days in the past month, or symptoms of pain and/or stiffness in or around hips, knees, ankles, feet, or lower back on most days of at least one month in the past six months; no current participation in a structured physical activity or weight management program and engaging in less than 150 min of moderate to vigorous physical activity weekly; self-reported height and weight consistent with a BMI (body mass index) of $25-50 \mathrm{~kg} / \mathrm{m}^{2}$ (confirmed at the baseline visit); aged 60 years or older; and ability to participate in study procedures, measurements, and interventions. Exclusion criteria included a score of 3 or more on the nine-item Mini Mental Status Questionnaire [24]; uncomplicated hip or knee surgery within the past 6 months; surgery with complications within the past year; plans for hip or knee surgery within the next year; steroid injections in either hip or knee in the past three months; diagnosis of rheumatoid arthritis, uncontrolled diabetes, or other health conditions that might interfere with exercise. The Exercise and Screening for You (EASY) screener was also administered to subjects to identify health reasons that may contraindicate participation in physical activity programs. Individuals who identified one or more high-risk condition on the EASY screener were required to obtain approval from their physician before participating. Four hundred and thirteen individuals completed baseline assessments and were enrolled in the trial.

\subsection{Study Measures}

Habitual dietary intake: All study subjects completed the semi-quantitative 110-item Block 2005 Food Frequency Questionnaire (FFQ) at baseline. The FFQ was administered by trained research staff and facilitated using a standardized portion guide. The FFQ was designed to assess habitual dietary intake of foods over the past year and current use of a variety of common dietary supplements (e.g., multivitamins, calcium, iron). Nutrient values that were calculated from the FFQ included: mean energy intake and percent of energy intake from fat, mean intake of fiber per 1000 calories, and mean intake of total fat, saturated fat, monounsaturated fat, polyunsaturated fat, trans fat, carbohydrates, protein, total fiber, sugar, cholesterol, sodium, calcium, vitamin D, vitamin C, iron, and vitamin B12. NutritionQuest (Berkeley, CA, USA) processed the FFQ survey booklets using a proprietary nutrient database developed from the USDA Food and Nutrient Database for Dietary Studies (FNDDS), version 1.0 [25] to obtain mean daily intake of macro- and micronutrients and HEI-2010 total and component scores. Only plausible (i.e., consuming 500-5000 calories daily) [26] and valid (i.e., $<10$ missing responses) [27] surveys were used for the analysis ( $n=13$ were excluded).

HEI-2010 is a commonly used measure of diet quality that assesses adherence to the 2010 DGAs [28]. HEI-2010 consists of 12 diet components that encourage adequacy (nine components) and moderation (three components) of foods and nutrients summing from $0-100$ points. The nine adequacy components are total fruit, whole fruit, total vegetables, greens and beans, whole grains, dairy, total protein foods, seafood and plant proteins, and fatty acids. The three moderation components are refined grains, sodium, and empty calories (calories from solid fats, alcoholic beverages, and added sugars). Higher scores in all components indicate closer conformance to the DGAs. Thus, higher intakes in the adequacy components receive higher scores and higher intakes in the moderation components receive lower scores. HEI-2010 uses an energy density approach to scoring (i.e., per 1000 calories or as a percent of calories), making it unnecessary to control for mean energy intake during statistical analysis [28]. 
Sociodemographic characteristics: A sociodemographic survey was administered to examine subjects' self-reported gender, age, race/ethnicity, educational attainment, relationship status, household income, health insurance, and current employment status.

Anthropometric measures: Body weight was measured twice using a digital scale (Tanita, Arlington Heights, IL, USA). Height was measured twice using a portable stadiometer (seca, Birmingham, UK). BMI was calculated from weight $(\mathrm{kg})$ and height $\left(\mathrm{m}^{2}\right)$ and was used to classify subjects into the categories of overweight $\left(25-29.9 \mathrm{~kg} / \mathrm{m}^{2}\right)$, obesity class I (30-34.9 kg/m²), obesity class II (35-39.9 kg/m²), or obesity class III $\left(\geq 40 \mathrm{~kg} / \mathrm{m}^{2}\right)$.

Osteoarthritis severity: The Western Ontario McMaster Universities Osteoarthritis Index (WOMAC) was used to evaluate symptoms of lower extremity OA in the study subjects. The WOMAC evaluates symptoms of pain (five items; score range 0-20), stiffness (two items; score range 0-8), and physical function (17 items; score range 0-68) for a total score of 0-96. A higher score reflects greater difficulties related to OA [29]. The validity of the WOMAC in patients with hip or knee OA has been demonstrated [29].

\subsection{Statistical Analysis}

Of the 413 participants in the trial, 13 had diet records with implausible estimates of energy intake ( $<500$ or $>5000$ calories). These participants were excluded from all analyses described here $(n=400)$. We used $t$-tests with pooled variance to test for differences in the HEI total score by participant demographics, BMI category (overweight/obese), and WOMAC global score (dichotomized at the median for the sample). We explored potential predictors of HEI scores using linear models with the HEI total score or a component score as the dependent variable and demographic variables, BMI, and the WOMAC global score as covariates. In these models, BMI and the WOMAC global score were entered as continuous variables. All analyses were conducted using SAS v 9.4.

\section{Results}

Four hundred and thirteen participants were recruited, and 400 had what were deemed plausible and valid FFQs and were included in the analysis. The sociodemographic and health characteristics of the participants are reported in Table 1. The majority of the sample was female and African American. The mean age of the participants was 67.8 years $(S D=5.9)$. Eighty percent of all subjects reported having completed some college or technical school or having a college degree; the median household income was $\$ 25,000$. Just over a quarter reported being married or a member of an unmarried couple.

The mean BMI at baseline was $34.8 \mathrm{~kg} / \mathrm{m}^{2}(\mathrm{SD}=5.5)$, with $45 \%$ classified as obesity class II and III. Based on the WOMAC, subjects reported having a moderate amount of OA-related symptoms.

The habitual nutrient intake of the participants by gender along with recommended intake targets is reported in Table 2. The mean energy intake for men and women was $1693 \mathrm{kcals}(\mathrm{SD}=702)$ and $1560 \mathrm{kcals}(\mathrm{SD}=711)$, respectively. The percent of energy consumed as total fat was similar for men and women at $39.8 \%$ and $39.9 \%$, respectively, exceeding daily recommendations. The mean intake of dietary fiber intake per 1000 calories was $8.8 \mathrm{~g}$ and $10.1 \mathrm{~g}$ for men and women, respectively. Mean values for sodium were above and for calcium and vitamin $\mathrm{D}$ were below daily recommendations for both men and women [30-32].

Table 3 presents subjects' HEI-2010 mean total and component scores by gender and categorizes their diet quality into three categories: "poor" (score range 0-50), "needs improvement" (score range 51-80), and "good" (score range 81-100) [33]. The overall diet quality needed improvement for both men and women, as indicated by the mean total scores of 64.3 and 66.6, respectively. More men $(12.3 \%)$ compared to women $(7.3 \%)$ had "poor" diet quality. Of the adequacy components, whole grains and dairy were particularly low, with mean scores of 4.4 (both genders) and 4.0 and 3.9 out of 10 for men and women, respectively. The remaining adequacy components received higher scores; notably, total protein foods had the greatest scores of 4.9 and 4.7 out of 5 for men and women, respectively. Of the three moderation components, refined grains received scores of 9.1 and 9.0 out of 10 for men and 
women, respectively, indicating relative compliance with recommendations, and for empty calories the scores were 11.4 and 12.4 out of 20 for men and women, respectively, indicating only modest compliance. The final moderation component, sodium, received scores of 4.8 and 4.4 out of 10 for men and women, respectively, indicating poor compliance with this recommendation. The men had notably lower component scores for total vegetables, greens and beans, and empty calories compared to the women.

Table 1. Characteristics of urban overweight and obese primarily African American older adults with osteoarthritis $(n=400)$.

\begin{tabular}{|c|c|c|}
\hline & Mean or $\%$ & SD or $n$ \\
\hline Age, years & 67.8 & 5.9 \\
\hline $60-69$ & $70 \%$ & 279 \\
\hline$\geq 70$ & $30 \%$ & 121 \\
\hline \multicolumn{3}{|l|}{ Gender } \\
\hline Female & $86 \%$ & 343 \\
\hline Male & $14 \%$ & 57 \\
\hline \multicolumn{3}{|l|}{ Race } \\
\hline Black or African-American, not Hispanic & $92 \%$ & 367 \\
\hline White, not Hispanic & $4 \%$ & 17 \\
\hline Hispanic & $1 \%$ & 5 \\
\hline Multiracial/other & $3 \%$ & 11 \\
\hline Education, years & 14.2 & 1.9 \\
\hline Not HS graduate, $n(\%)$ & $6 \%$ & 22 \\
\hline HS graduate/GED & $15 \%$ & 61 \\
\hline Some college or technical school & $43 \%$ & 170 \\
\hline College graduate & $37 \%$ & 147 \\
\hline Employed full or part-time & $13 \%$ & 53 \\
\hline \multicolumn{3}{|l|}{ Relationship status } \\
\hline Married or member of unmarried couple & $26 \%$ & 104 \\
\hline Divorced or separated & $36 \%$ & 145 \\
\hline Widowed & $22 \%$ & 88 \\
\hline Never married & $16 \%$ & 63 \\
\hline Income, median $(n=338)$ & 25,000 & \\
\hline \multicolumn{3}{|l|}{ Health insurance $^{\text {a }}$} \\
\hline Medicare & $68 \%$ & 271 \\
\hline Medicaid & $17 \%$ & 68 \\
\hline Private/supplemental & $42 \%$ & 166 \\
\hline WOMAC global score $(0-96)^{b}$ & 26.7 & 17.2 \\
\hline Pain subscale (0-20) & 5.6 & 4.0 \\
\hline Stiffness subscale (0-8) & 3.2 & 1.7 \\
\hline Physical functioning subscale (0-68) & 17.9 & 12.7 \\
\hline BMI, $\mathrm{kg} / \mathrm{m}^{2}$ & 34.8 & 5.5 \\
\hline \multicolumn{3}{|l|}{ BMI category } \\
\hline Overweight $\left(25-29.9 \mathrm{~kg} / \mathrm{m}^{2}\right)$ & $20 \%$ & 78 \\
\hline Obesity class I $\left(30-34.9 \mathrm{~kg} / \mathrm{m}^{2}\right)$ & $36 \%$ & 144 \\
\hline Obesity class II (35-39.9 kg/m²) & $25 \%$ & 100 \\
\hline Obesity class III $\left(\geq 40 \mathrm{~kg} / \mathrm{m}^{2}\right)$ & $20 \%$ & 78 \\
\hline
\end{tabular}

a Percentage of participants reporting each type of insurance; some participants reported more than one type of insurance; ${ }^{\mathrm{b}} \mathrm{A}$ higher score indicates greater difficulties due to osteoarthritis (OA). Abbreviations: BMI, body mass index; GED, General Educational Development; HS, high school; WOMAC, Western Ontario McMaster Universities Osteoarthritis Index. 
Table 2. Habitual nutrient intake of urban overweight and obese primarily African American older adults with osteoarthritis $(n=400)$.

\begin{tabular}{|c|c|c|c|c|c|c|c|c|}
\hline \multirow{3}{*}{ Variable } & \multicolumn{4}{|c|}{ Intake } & \multicolumn{4}{|c|}{ Recommendation } \\
\hline & \multicolumn{2}{|c|}{ Men $(n=57)$} & \multicolumn{2}{|c|}{ Women $(n=343)$} & \multicolumn{2}{|c|}{ Men } & \multicolumn{2}{|c|}{ Women } \\
\hline & Mean & SD & Mean & SD & 51-70 Years & $>70$ Years & 51-70 Years & $>70$ Years \\
\hline Energy, kcal [34] & 1693 & 702 & 1560 & 711 & $2000-2200$ & 2000 & 1600 & 1600 \\
\hline Total fat, g [34] & 75.1 & 34.1 & 70.0 & 35.6 & Not determined & Not determined & Not determined & Not determined \\
\hline Fat, \% calories [34] & 39.8 & 5.9 & 39.9 & 7.1 & $20-35$ & $20-35$ & $20-35$ & $20-35$ \\
\hline Carbohydrates, g [34] & 189.7 & 86.6 & 178.5 & 82.0 & 130 & 130 & 130 & 130 \\
\hline Protein, g [34] & 65.0 & 30.5 & 59.4 & 31.8 & 56 & 56 & 46 & 46 \\
\hline Fiber, g [34] & 14.2 & 6.2 & 15.3 & 7.9 & 30 & 30 & 21 & 21 \\
\hline Fiber, g/1000 kcal & 8.8 & 3.0 & 10.1 & 3.7 & 14 & 14 & 14 & 14 \\
\hline Saturated fat, g [32] & 22.1 & 11.8 & 19.9 & 10.7 & $<10 \%$ of intake & $<10 \%$ of intake & $<10 \%$ of intake & $<10 \%$ of intake \\
\hline Monounsaturated fat, $g$ [35] & 30.4 & 14.2 & 28.0 & 14.3 & Majority of fats & Majority of fats & Majority of fats & Majority of fats \\
\hline Polyunsaturated fat, g [36] & 16.8 & 7.0 & 16.7 & 9.0 & Majority of fats & Majority of fats & Majority of fats & Majority of fats \\
\hline Trans fats, $g$ [32] & 2.3 & 1.6 & 2.0 & 1.2 & As low as possible & As low as possible & As low as possible & As low as possible \\
\hline Sugars, g [32] & 94.9 & 55.1 & 88.9 & 48.6 & $<10 \%$ of energy & $<10 \%$ of energy & $<10 \%$ of energy & $<10 \%$ of energy \\
\hline Cholesterol, mg [34] & 282.2 & 193.5 & 227.7 & 147.0 & As low as possible & As low as possible & As low as possible & As low as possible \\
\hline Sodium, mg [32] & 2673 & 1212 & 2524 & 1274 & $<2300$ & $<2300$ & $<2300$ & $<2300$ \\
\hline Calcium, mg [30] & 632.1 & 386.0 & 586.3 & 296.8 & 1000 & 1200 & 1200 & 1200 \\
\hline Vitamin D, IU [30] & 120.7 & 84.8 & 90.7 & 76.1 & 600 & 800 & 600 & 800 \\
\hline Vitamin C, mg [37] & 94.0 & 67.1 & 100.6 & 62.8 & 90 & 90 & 75 & 75 \\
\hline Iron, mg [31] & 11.7 & 4.7 & 10.9 & 5.5 & 8 & 8 & 8 & 8 \\
\hline Vitamin B12, mcg [38] & 4.7 & 2.8 & 3.8 & 2.8 & 2.4 & 2.4 & 2.4 & 2.4 \\
\hline
\end{tabular}

Abbreviation: SD, standard deviation. 
There was no significant difference in the HEI-2010 total score based on sex, race, education, relationship status, or employment (Table 4). However, significant differences were observed based on age, BMI, and WOMAC global score. Specifically, a lower HEI-2010 total score was reported for subjects less than 70 years old compared to those $\geq 70$ years old ( 65.4 vs. $68.4 ; p=0.009$ ), obese compared to overweight $(65.7 \mathrm{vs.} 68.8 ; p=0.02)$, and for those with a WOMAC global score greater than or equal to the median compared to those with a global score less than the median (65.2 vs. 67.4; $p=0.04)$.

Table 3. Healthy Eating Index-2010 (HEI-2010) total and component scores of urban overweight and obese primarily African American older adults with osteoarthritis $(n=400)$.

\begin{tabular}{lcccc}
\hline & \multicolumn{2}{c}{ Men $(\boldsymbol{n}=\mathbf{5 7})$} & \multicolumn{2}{c}{ Women $(\boldsymbol{n}=\mathbf{3 4 3})$} \\
\cline { 2 - 5 } & Mean or \% & SD or $\boldsymbol{N}$ & Mean or \% & SD or $\boldsymbol{N}$ \\
\hline Healthy Eating Index-2010 (0-100) & 64.3 & 10.7 & 66.6 & 10.4 \\
Total fruit (0-5) & 3.5 & 1.5 & 3.7 & 1.4 \\
Whole fruit (0-5) & 3.6 & 1.5 & 3.9 & 1.4 \\
Total vegetables (0-5) & 3.4 & 1.3 & 4.1 & 1.1 \\
Greens and beans (0-5) & 3.8 & 1.5 & 4.3 & 1.3 \\
Whole grains (0-10) & 4.4 & 3.0 & 4.4 & 2.9 \\
Dairy (0-10) & 4.0 & 2.1 & 3.9 & 2.3 \\
Total protein foods (0-5) & 4.9 & 0.4 & 4.7 & 0.7 \\
Seafood and plant protein (0-5) & 4.1 & 1.3 & 4.2 & 1.2 \\
Fatty acids (0-10) & 7.3 & 2.5 & 7.5 & 2.4 \\
Refined grains (0-10) & 9.1 & 1.3 & 9.0 & 1.6 \\
Sodium (0-10) & 4.8 & 2.8 & 4.4 & 3.0 \\
Empty calories (0-20) & 11.4 & 5.3 & 12.4 & 4.5 \\
HEI-2010 category & & & & \\
$\quad$ Poor (0-50) & $12.3 \%$ & 7 & $7.3 \%$ & 25 \\
$\quad$ Needs improvement (51-80) & $82.5 \%$ & 47 & $86.0 \%$ & 295 \\
$\quad$ Good (81-100) & $5.3 \%$ & 3 & $6.7 \%$ & 23 \\
\hline
\end{tabular}

Abbreviation: SD, standard deviation.

Table 4. Healthy Eating Index-2010 total score by subject characteristics.

\begin{tabular}{lcccc}
\hline & $n$ & Mean & SD & $p^{\text {a }}$ \\
\hline $\begin{array}{l}\text { Sex } \\
\quad \text { Female }\end{array}$ & 343 & 66.6 & 10.4 & 0.13 \\
$\quad$ Male & 57 & 64.3 & 10.7 & \\
\hline Age, years & & & & 0.009 \\
\hline$\quad 60-69$ & 279 & 65.4 & 10.3 & \\
$\quad 121$ & 68.4 & 10.6 & \\
$\quad$ Race & & & & 0.55 \\
\hline African-American & 367 & 66.2 & 10.4 & \\
$\quad$ Other & 33 & 67.4 & 11.6 & \\
\hline Education & & & & 0.15 \\
\hline$\quad$ College graduate & 147 & 67.3 & 10.7 & \\
$\quad$ Not college graduate & 253 & 65.7 & 10.3 & \\
\hline Relationship status & & & & 0.80 \\
\hline$\quad$ Married or unmarried couple & 104 & 66.1 & 10.5 & \\
$\quad$ Other & 296 & 66.4 & 10.5 & \\
\hline
\end{tabular}


Table 4. Cont

\begin{tabular}{lcccc}
\hline & $n$ & Mean & SD & $p^{\text {a }}$ \\
\hline Employment & & & & 0.07 \\
\hline Full or part-time & 53 & 68.7 & 10.2 & \\
$\quad$ Not employed & 347 & 65.9 & 10.5 & \\
\hline BMI, kg/m & & & & 0.02 \\
\hline$\quad$ Overweight (25-29.9) & 78 & 68.8 & 9.2 & \\
$\quad$ Obese ( $\geq 30)$ & 322 & 65.7 & 10.7 & \\
\hline WOMAC global score & & & & 0.04 \\
\hline$<24$ (median) & 199 & 67.4 & 10.5 & \\
$\geq 24$ & 201 & 65.2 & 10.4 & \\
\hline
\end{tabular}

${ }^{\text {a }}$ From $t$-tests with pooled variance. Abbreviations: BMI, body mass index; WOMAC, Western Ontario McMaster Universities Osteoarthritis Index.

The sociodemographic and health-related characteristics were tested as possible predictors of HEI-2010 total as well as HEI-2010 component scores in multivariable linear regression models (Table 5). Age $(\beta=0.21$ (SE 0.09)), employment $(\beta=3.26$ (SE 1.56)), and BMI $(\beta=-0.20$ (SE 0.10)) were independent predictors of the HEI-2010 total score when adjusting for the other sociodemographic and health-related variables. When examining the HEI-2010 component scores, male gender was a significant inverse predictor of total vegetable score $(\beta=-0.70$ (SE 0.17)), and, greens and beans score $(\beta=-0.53$ (SE 0.17)), and a positive predictor of total protein score $(\beta=0.22$ (SE 0.09)) when adjusting for the other sociodemographic and health-related variables. Age was a significant positive predictor of total fruit score $(\beta=0.03$ (SE 0.01)) and whole fruit score $(\beta=0.03$ (SE 0.01)). College graduate education level was a positive predictor of total fruit score $(\beta=0.34$ (SE 0.15)) and surprisingly a negative predictor of whole grain score $(\beta=-0.76$ (SE 0.30)). Race other than African American was a significant positive predictor of whole grain score $(\beta=1.17$ (SE 0.53$))$ and dairy score $(\beta=1.49$ (SE 0.42)) as well as a negative predictor of refined grain score ( $\beta=-1.22$ (SE 0.28)). WOMAC global score was a positive predictor of dairy score $(\beta=0.02$ (SE 0.01)) and a negative predictor of fatty acids score $(\beta=-0.02$ (SE 0.01)). Finally, full or part-time employment was a positive predictor of empty calories score $(\beta=1.41$ (SE 0.69)). 
Table 5. Predictors of Healthy Eating Index-2010 (HEI-2010) total and component scores in urban overweight and obese primarily African American older adults with osteoarthritis.

\begin{tabular}{|c|c|c|c|c|c|c|c|c|c|c|c|c|c|c|c|}
\hline & \multicolumn{3}{|c|}{ HEI-2010 $\left(R^{2}=0.06\right)$} & \multicolumn{3}{|c|}{ Total Fruit $\left(R^{2}=0.06\right)$} & \multicolumn{3}{|c|}{ Whole Fruit $\left(R^{2}=0.05\right)$} & \multicolumn{3}{|c|}{ Total Vegetables $\left(R^{2}=0.07\right)$} & \multicolumn{3}{|c|}{ Empty Calories $\left(R^{2}=0.06\right)$} \\
\hline & $\mathbf{b}$ & SE & $p$ & $\mathbf{b}$ & SE & $p$ & $\mathbf{b}$ & SE & $p$ & $\mathbf{b}$ & $\mathrm{SE}$ & $p$ & $\mathbf{b}$ & SE & $p$ \\
\hline Intercept & 59.91 & 7.67 & $<0.001$ & 2.37 & 1.06 & 0.03 & 2.38 & 1.05 & 0.02 & 3.72 & 0.85 & $<0.001$ & 9.08 & 3.42 & 0.008 \\
\hline Male & -2.56 & 1.53 & 0.10 & -0.19 & 0.21 & 0.38 & -0.30 & 0.21 & 0.15 & -0.70 & 0.17 & $<0.001$ & -0.87 & 0.68 & 0.20 \\
\hline Age, years & 0.21 & 0.09 & 0.02 & 0.03 & 0.01 & 0.01 & 0.03 & 0.01 & 0.02 & 0.01 & 0.01 & 0.45 & 0.08 & 0.04 & 0.05 \\
\hline Other race (not African-American) & 0.93 & 1.90 & 0.62 & -0.43 & 0.26 & 0.10 & -0.12 & 0.26 & 0.64 & 0.06 & 0.21 & 0.79 & 1.37 & 0.85 & 0.11 \\
\hline College graduate & 0.95 & 1.09 & 0.39 & 0.34 & 0.15 & 0.03 & 0.29 & 0.15 & 0.06 & 0.24 & 0.12 & 0.05 & 0.87 & 0.49 & 0.07 \\
\hline Married or unmarried couple & -0.56 & 1.21 & 0.64 & 0.11 & 0.17 & 0.50 & -0.02 & 0.17 & 0.91 & 0.10 & 0.13 & 0.45 & -0.71 & 0.54 & 0.19 \\
\hline Employed full or part-time & 3.26 & 1.56 & 0.04 & 0.30 & 0.22 & 0.16 & 0.40 & 0.21 & 0.06 & 0.10 & 0.17 & 0.58 & 1.41 & 0.69 & 0.04 \\
\hline BMI, $\mathrm{kg} / \mathrm{m}^{2}$ & -0.20 & 0.10 & 0.04 & -0.02 & 0.01 & 0.10 & -0.01 & 0.01 & 0.37 & 0.00 & 0.01 & 0.82 & -0.06 & 0.04 & 0.19 \\
\hline \multirow[t]{3}{*}{ WOMAC global score ${ }^{a}$} & -0.04 & 0.03 & 0.16 & -0.01 & 0.00 & 0.09 & -0.01 & 0.00 & 0.08 & -0.01 & 0.00 & 0.06 & -0.02 & 0.01 & 0.17 \\
\hline & \multicolumn{3}{|c|}{ Greens and Beans $\left(R^{2}=0.03\right)$} & \multicolumn{3}{|c|}{ Whole Grains $\left(R^{2}=0.03\right)$} & \multicolumn{3}{|c|}{ Dairy $\left(R^{2}=0.06\right)$} & \multicolumn{3}{|c|}{$\begin{array}{l}\text { Total Protein Foods } \\
\left(\mathbf{R}^{2}=0.03\right)\end{array}$} & & & \\
\hline & b & SE & $p$ & $\mathbf{b}$ & SE & $p$ & $\mathbf{b}$ & SE & $p$ & $\mathbf{b}$ & SE & $p$ & & & \\
\hline Intercept & 3.96 & 0.97 & $<0.001$ & 3.37 & 2.13 & 0.11 & 4.12 & 1.69 & 0.02 & 4.95 & 0.47 & $<0.001$ & & & \\
\hline Male & -0.53 & 0.19 & 0.006 & -0.15 & 0.42 & 0.73 & 0.08 & 0.34 & 0.82 & 0.22 & 0.09 & 0.02 & & & \\
\hline Age, years & 0.01 & 0.01 & 0.42 & 0.02 & 0.03 & 0.45 & 0.00 & 0.02 & 0.94 & -0.01 & 0.01 & 0.11 & & & \\
\hline Race not African-American & 0.03 & 0.24 & 0.92 & 1.17 & 0.53 & 0.03 & 1.49 & 0.42 & $<0.001$ & -0.11 & 0.12 & 0.34 & & & \\
\hline College graduate & 0.08 & 0.14 & 0.54 & -0.76 & 0.30 & 0.01 & -0.30 & 0.24 & 0.21 & 0.08 & 0.07 & 0.24 & & & \\
\hline Married or unmarried couple & 0.24 & 0.15 & 0.12 & -0.34 & 0.34 & 0.31 & -0.48 & 0.27 & 0.07 & 0.00 & 0.07 & 0.99 & & & \\
\hline Employed full or part-time & 0.08 & 0.20 & 0.69 & 0.52 & 0.43 & 0.23 & -0.32 & 0.34 & 0.35 & 0.06 & 0.10 & 0.50 & & & \\
\hline BMI, $\mathrm{kg} / \mathrm{m}^{2}$ & -0.01 & 0.01 & 0.43 & 0.00 & 0.03 & 0.90 & -0.01 & 0.02 & 0.58 & 0.01 & 0.01 & 0.16 & & & \\
\hline \multirow[t]{3}{*}{ WOMAC global score $^{\text {a }}$} & 0.00 & 0.00 & 0.77 & 0.00 & 0.01 & 0.78 & 0.02 & 0.01 & 0.01 & 0.00 & 0.00 & 0.66 & & & \\
\hline & \multicolumn{3}{|c|}{$\begin{array}{l}\text { Seafood and Plant Proteins } \\
\qquad\left(R^{2}=0.03\right)\end{array}$} & \multicolumn{3}{|c|}{ Fatty Acids $\left(R^{2}=0.04\right)$} & \multicolumn{3}{|c|}{ Refined Grains $\left(R^{2}=0.06\right)$} & \multicolumn{3}{|c|}{ Sodium $\left(R^{2}=0.02\right)$} & & & \\
\hline & $\mathbf{b}$ & SE & $p$ & $\mathbf{b}$ & SE & $p$ & $\mathbf{b}$ & SE & $p$ & $\mathbf{b}$ & SE & $p$ & & & \\
\hline Intercept & 3.46 & 0.90 & $<0.001$ & 8.19 & 1.79 & $<0.001$ & 9.85 & 1.14 & $<0.001$ & 4.45 & 2.22 & 0.046 & & & \\
\hline Male & -0.12 & 0.18 & 0.52 & -0.36 & 0.36 & 0.31 & 0.00 & 0.23 & 0.98 & 0.37 & 0.44 & 0.41 & & & \\
\hline Age, years & 0.02 & 0.01 & 0.13 & 0.01 & 0.02 & 0.71 & 0.00 & 0.01 & 0.78 & 0.01 & 0.03 & 0.57 & & & \\
\hline Race not African-American & 0.23 & 0.22 & 0.30 & -0.70 & 0.44 & 0.12 & -1.22 & 0.28 & $<0.001$ & -0.82 & 0.55 & 0.14 & & & \\
\hline College graduate & 0.23 & 0.13 & 0.08 & -0.10 & 0.26 & 0.69 & -0.17 & 0.16 & 0.28 & 0.16 & 0.32 & 0.61 & & & \\
\hline Married or unmarried couple & 0.05 & 0.14 & 0.75 & 0.25 & 0.28 & 0.39 & 0.16 & 0.18 & 0.37 & 0.09 & 0.35 & 0.80 & & & \\
\hline Employed full or part-time & 0.22 & 0.18 & 0.23 & 0.61 & 0.36 & 0.09 & 0.22 & 0.23 & 0.34 & -0.34 & 0.45 & 0.45 & & & \\
\hline BMI, $\mathrm{kg} / \mathrm{m}^{2}$ & -0.02 & 0.01 & 0.18 & -0.02 & 0.02 & 0.34 & -0.02 & 0.01 & 0.09 & -0.03 & 0.03 & 0.35 & & & \\
\hline WOMAC global score ${ }^{a}$ & 0.00 & 0.00 & 0.46 & -0.02 & 0.01 & 0.02 & 0.00 & 0.00 & 0.36 & 0.00 & 0.01 & 0.61 & & & \\
\hline
\end{tabular}

From linear regression models with HEI-2010 or components as the dependent variable and the variables shown as independent variables. $n=400$; records with estimated energy $<500$ or $>5000$ were excluded from the analysis $(n=13)$. ${ }^{a}$ A higher score indicates greater difficulties due to OA. Abbreviations: b, beta; BMI, body mass index; WOMAC, Western Ontario McMaster Universities Osteoarthritis Index. 


\section{Discussion}

Although weight loss has been shown to have a promising effect on OA progression, functional outcomes, and symptom management $[9,10]$, weight loss is difficult to achieve and maintain for most individuals, including African Americans [39]. Diet quality may be a more salient intervention target for managing obesity-related OA, given that it involves changing one's dietary pattern but not restricting calories. However, there is limited research examining diet quality and nutrient intake of overweight and obese African American older adults with OA, despite the fact that this population is disproportionately burdened by both chronic conditions [3-5]. From a public health and clinical perspective, information on diet quality is crucial to inform and target efforts addressing the nutritional needs and improvement of dietary intakes of this at-risk segment of the U.S. population [40]. To advance the literature, we conducted a cross-sectional analysis to describe the diet quality (HEI-2010) and nutrient intake of 400 urban overweight and obese primarily African American older adults with self-reported lower extremity OA.

Overall, we report that the diet quality of both men and women in our cohort needs improvement [33]. Our findings are consistent with the mean HEI-2010 total score reported for a representative sample of U.S. adults aged 65 years and older $(69.3(\mathrm{SD}=1.8))$ [41]. While no studies have examined associations between diet quality measured by HEI and OA risk, progression, or symptom management, one study reported that higher mean HEI-2005 total score was associated with better gait speed in U.S. older adults, a marker of physical function often compromised in those with OA [42]. Two studies examined associations between diet quality, defined as adherence to a Mediterranean diet [20] and dietary glycemic index [13], and OA-related outcomes. Higher adherence to a Mediterranean diet, a largely plant-based eating pattern including olive oil as the predominant fat, was associated with lower OA prevalence in the large North American OA Initiative cohort [20]. In another study of Korean adults, adherence to a diet with a low glycemic index, indicative of low consumption of refined carbohydrates, was associated with decreased risk of symptomatic knee OA in women [13]. The authors of both studies surmised that the positive OA outcomes were attributed to the anti-inflammatory [43,44], anti-oxidative [45,46], and cartilage preserving [47-49] effects of the higher quality diets. However, randomized controlled trials are needed to confirm these findings and to understand the underlying biological mechanisms linking diet quality and OA.

In our cohort, empty calories, whole grains, and dairy were among the lowest scoring of the 12 HEI-2010 components. Compared to HEI-2010 component scores reported for the general population of older U.S. adults ( $\geq 65$ years), our mean whole grains score was similar (4.4 (SD 2.9) vs. 4.23 (SD 0.34)), while empty calories (12.2 (SD 4.7) vs. 14.99 (SD 0.44)) and dairy scores (3.9 (SD 2.3) vs. 5.99 (SD 0.16)) were lower [41]. A lower dairy score was somewhat expected given the higher rates of perceived and actual lactose intolerance in African Americans [50]. Moreover, aligning with the same diet quality components, we also observed that mean nutrient intakes for fiber, calcium, and vitamin D were below recommendations, while the percentage of calories as total fat exceeded recommendations. Intake of empty calories, whole grains/fiber, and dairy may be relevant to OA. Specifically, "empty calories" from solid fats, added sugars, and alcohol [28] may contribute to unhealthy weight gain [51], increased production of pro-inflammatory cytokines and adipokines, and oxidative stress [45,52,53], all of which may play an important role in the mechanical and metabolic pathogenesis of OA $[7,8]$. Similarly, low intake of whole grains, which are rich in fiber and antioxidant nutrients, is associated with poor body weight regulation and increased systemic inflammation $[54,55]$. The association between dairy foods and OA is somewhat muddled. In a large cohort study of Australian adults, greater dairy consumption was associated with increased risk for hip replacement in men with hip OA [56]. Meanwhile, in a large North American cohort, higher milk consumption was associated with reduced OA progression in women [57]. Some argue that it is not dairy foods per se that are protective for OA but the nutrients they contain such as calcium, vitamin $\mathrm{D}$, and $\mathrm{B}$ vitamins, given their role in bone and muscle health [58-60]. Clearly, randomized dietary intervention trials are needed to corroborate observational findings and to understand the relationships between empty calories, 
whole grains/fiber, dairy, and OA. Given the observed inadequacy, these components of diet quality may be important intervention targets for older African American adults with OA.

We evaluated associations between diet quality and sociodemographic and health characteristics of the study participants. In the bivariate analyses, we found better diet quality in adults over 70 years old compared to those aged 60-69 years, in overweight subjects compared to obese, and in those with lower global scores on WOMAC compared to those with higher global scores. A study by Reedy et al. [61] of diet quality and all-cause, cardiovascular disease, and cancer mortality among older adults found that younger age and higher BMI were both significant inverse predictors of HEI-2010 total scores. In other studies examining predictors of diet quality in U.S. adults, female gender, non-Hispanic white race, and higher perceived social support among men were significant predictors of superior diet quality based on HEI, while living alone was associated with lower diet quality $[40,62,63]$. To our knowledge, existing studies have not evaluated associations between HEI-2010 and WOMAC global scores. However, in the study by Veronese and colleagues, greater adherence to a Mediterranean diet was associated with lower WOMAC global scores among adults with OA [20], suggesting that OA severity (i.e., pain, stiffness, and overall physical function) is linked with diet quality. However, confirmation in randomized controlled trials is needed.

We also examined associations between the HEI-2010 component scores and sociodemographic and health characteristics of the participants. Older age was associated with higher total fruit and whole fruit scores; male gender was associated with lower total vegetables and greens and beans scores, as well as higher total protein score; self-identifying race as other than African American was associated with higher whole grains and dairy scores and lower refined grains score; full or part-time employment was associated with higher empty calories score; and a higher WOMAC global score was associated with lower fatty acid score and higher dairy score. Partially corroborating our findings, in a representative sample of U.S. adults, older age was associated with higher intake of total and whole fruits; male gender was associated with lower intake of vegetables including greens and beans; and identifying as non-Hispanic black was associated with lower whole grain intake [64-66]. Although no studies have examined associations between WOMAC global score and HEI components, higher intake of saturated vs. unsaturated fatty acids is associated with greater systemic inflammation and progression of lower extremity OA [67], providing some biological plausibility to our observed association.

A major strength of our study was the assessment of diet quality using HEI-2010 and the examination of sociodemographic and health predictors of diet quality in a majority urban African American sample of overweight and obese older adults with lower extremity OA. Dietary behaviors of racial/ethnic minority older adults have been largely understudied despite disparities in several diet-related chronic diseases and the importance of this data to designing appropriate interventions. However, this study is not without limitations. First, we acknowledge that there may be many other factors that contribute significantly to diet quality and nutrient intake of older adults that were not addressed, particularly concerning racial/ethnic minorities living in urban areas, including food-access, food security, the physical environment, living conditions, real and perceived discrimination, social support, and cohesion $[40,63,68,69]$. Future studies should examine how these factors relate to diet quality in this subgroup. Second, an FFQ is biased by poor participant recall of dietary intake and by participant's provision of socially desirable responses [66]. Further, underreporting of food intake has been observed in obese individuals as well as African American women [70-72]. Our sample size may have limited our ability to effectively examine diet patterns for subgroups (e.g., men vs. women). Because the analysis was cross-sectional, we were also not able to infer causal associations between diet quality and the sociodemographic and health factors that we examined. Lastly, it is important to acknowledge that generalizability of our findings may be limited given that the individuals that we assessed were enrolling in a lifestyle intervention program and thus may not be representative of the wider population of urban overweight and obese older African Americans adults with lower extremity OA. 


\section{Conclusions}

Diet quality and components of diet quality including empty calories, whole grains/fiber, and dairy may play an important role in OA risk, progression, and symptom management. Still, well-designed randomized dietary intervention trials are needed to provide conclusive evidence for the role of diet quality in OA-related outcomes. In the current study of urban, primarily African American overweight and obese older adults with lower extremity OA, overall diet quality (HEI-2010) and component scores for empty calories, whole grains, and dairy needed improvement, and intakes of several nutrients including vitamin D, calcium, and fiber did not meet sex- and age-specific recommendations. Our findings can be used to inform future dietary intervention trials and public health messaging for this at-risk group.

Acknowledgments: This research was supported by R01AG039374 from the National Institute on Aging (S.H., M.F.), the American Cancer Society of Illinois grant \#2617755 (L.T.-H.) and American Cancer Society Mentored Research Scholar grant MRSG014-025-01-CNE (L.T.-H.). The authors thank P.J. Desai, Amy Shah, Sarah Olender, and Mirjana Antonic for their research and administrative support. We would also like to thank the study participants for giving their time generously to the project.

Author Contributions: L.T.-H., S.V., M.F., and S.H. conceived and designed the study. L.S. performed the data analysis. S.V., L.T.-H., and T.W. wrote the majority of the paper, with all authors reviewing and approving the manuscript.

Conflicts of Interest: The authors declare no conflict of interest.

\section{References}

1. United States Census Bereau. The Nation's Older Population Is Still Growing, Census Bureau Reports. 2017. Available online: https:/ / www.census.gov/newsroom/press-releases/2017/cb17-100.html (accessed on 30 January 2018).

2. Niccoli, T.; Partridge, L. Ageing as a risk factor for disease. Curr. Biol. 2012, 22, R741-R752. [CrossRef] [PubMed]

3. Jordan, J.M. An ongoing assessment of osteoarthritis in African Americans and Caucasians in North Carolina: The Johnston County osteoarthritis project. Trans. Am. Clin. Climatol. Assoc. 2015, 126, 77-86. [PubMed]

4. Dillon, C.F.; Rasch, E.K.; Gu, Q.; Hirsch, R. Prevalence of knee osteoarthritis in the united states: Arthritis data from the third national health and nutrition examination survey 1991-94. J. Rheumatol. 2006, 33, 2271-2279. [PubMed]

5. Flegal, K.M.; Kruszon-Moran, D.; Carroll, M.D.; Fryar, C.D.; Ogden, C.L. Trends in obesity among adults in the united states, 2005 to 2014. JAMA 2016, 315, 2284-2291. [CrossRef] [PubMed]

6. Barbour, K.E.; Helmick, C.G.; Boring, M.; Qin, J.; Pan, L.; Hootman, J.M. Obesity trends among us adults with doctor-diagnosed arthritis 2009-2014. Arthritis Care Res. (Hoboken) 2017, 69, 376-383. [CrossRef] [PubMed]

7. Yusuf, E. Metabolic factors in osteoarthritis: Obese people do not walk on their hands. Arthritis Res. Ther. 2012, 14, 123. [CrossRef] [PubMed]

8. Robinson, W.H.; Lepus, C.M.; Wang, Q.; Raghu, H.; Mao, R.; Lindstrom, T.M.; Sokolove, J. Low-grade inflammation as a key mediator of the pathogenesis of osteoarthritis. Nat. Rev. Rheumatol. 2016, 12, 580-592. [CrossRef] [PubMed]

9. Beavers, K.M.; Beavers, D.P.; Newman, J.J.; Anderson, A.M.; Loeser, R.F., Jr.; Nicklas, B.J.; Lyles, M.F.; Miller, G.D.; Mihalko, S.L.; Messier, S.P. Effects of total and regional fat loss on plasma CRP and Il-6 in overweight and obese, older adults with knee osteoarthritis. Osteoarthr. Cartil. 2015, 23, 249-256. [CrossRef] [PubMed]

10. Messier, S.P.; Mihalko, S.L.; Legault, C.; Miller, G.D.; Nicklas, B.J.; DeVita, P.; Beavers, D.P.; Hunter, D.J.; Lyles, M.F.; Eckstein, F.; et al. Effects of intensive diet and exercise on knee joint loads, inflammation, and clinical outcomes among overweight and obese adults with knee osteoarthritis: The idea randomized clinical trial. JAMA 2013, 310, 1263-1273. [CrossRef] [PubMed]

11. Wluka, A.E.; Lombard, C.B.; Cicuttini, F.M. Tackling obesity in knee osteoarthritis. Nat. Rev. Rheumatol. 2013, 9, 225-235. [CrossRef] [PubMed] 
12. Henriksen, M.; Christensen, R.; Danneskiold-Samsoe, B.; Bliddal, H. Changes in lower extremity muscle mass and muscle strength after weight loss in obese patients with knee osteoarthritis: A prospective cohort study. Arthritis Rheum. 2012, 64, 438-442. [CrossRef] [PubMed]

13. So, M.W.; Lee, S.; Kim, S.H. Association between dietary glycemic index and knee osteoarthritis: The Korean national health and nutrition examination survey 2010-2012. J. Acad. Nutr. Diet. 2018. [CrossRef] [PubMed]

14. Wirt, A.; Collins, C.E. Diet quality-What is it and does it matter? Public Health Nutr. 2009, 12, $2473-2492$. [CrossRef] [PubMed]

15. Kennedy, E.T.; Ohls, J.; Carlson, S.; Fleming, K. The healthy eating index: Design and applications. J. Am. Diet. Assoc. 1995, 95, 1103-1108. [CrossRef]

16. Guenther, P.M.; Kirkpatrick, S.I.; Reedy, J.; Krebs-Smith, S.M.; Buckman, D.W.; Dodd, K.W.; Casavale, K.O.; Carroll, R.J. The healthy eating index-2010 is a valid and reliable measure of diet quality according to the 2010 dietary guidelines for Americans. J. Nutr. 2014, 144, 399-407. [CrossRef] [PubMed]

17. Fung, T.T.; Pan, A.; Hou, T.; Chiuve, S.E.; Tobias, D.K.; Mozaffarian, D.; Willett, W.C.; Hu, F.B. Long-term change in diet quality is associated with body weight change in men and women. J. Nutr. 2015, 145, 1850-1856. [CrossRef] [PubMed]

18. Anderson, C.; Harrigan, M.; George, S.M.; Ferrucci, L.M.; Sanft, T.; Irwin, M.L.; Cartmel, B. Changes in diet quality in a randomized weight loss trial in breast cancer survivors: The lifestyle, exercise, and nutrition (LEAN) study. NPJ Breast Cancer 2016, 2, 16026. [CrossRef] [PubMed]

19. Mohammadshahi, M.; Haidari, F.; Karandish, M.; Ebrahimi, S.; Haghighizadeh, M.-H. A randomized clinical trial of nutrition education for improvement of diet quality and inflammation in Iranian obese women. J. Nutr. Metab. 2014, 2014, 605782. [CrossRef] [PubMed]

20. Veronese, N.; Stubbs, B.; Noale, M.; Solmi, M.; Luchini, C.; Smith, T.O.; Cooper, C.; Guglielmi, G.; Reginster, J.-Y.; Rizzoli, R.; et al. Adherence to a mediterranean diet is associated with lower prevalence of osteoarthritis: Data from the osteoarthritis initiative. Clin. Nutr. 2017, 36, 1609-1614. [CrossRef] [PubMed]

21. Wang, Y.; Chen, X. Between-group differences in nutrition- and health-related psychosocial factors among us adults and their associations with diet, exercise, and weight status. J. Acad. Nutr. Diet. 2012, 112, 486-498. [CrossRef] [PubMed]

22. Juan, W.Y.G.; Patricia, M.; Kott, P.S. Diet quality of older Americans in 1994-1996 and 2001-2002 as measured by the healthy eating index-2005. In Nutrition Insight 41; USDA Center for Nutrition Policy and Promotion: Alexandria, VA, USA, 2008.

23. Smith-Ray, R.L.; Fitzgibbon, M.L.; Tussing-Humphreys, L.; Schiffer, L.; Shah, A.; Huber, G.M.; Braunschweig, C.; Campbell, R.T.; Hughes, S.L. Fit and strong! Plus: Design of a comparative effectiveness evaluation of a weight management program for older adults with osteoarthritis. Contemp. Clin. Trials 2014, 37, 178-188. [CrossRef] [PubMed]

24. Pfeiffer, E. A short portable mental status questionnaire for the assessment of organic brain deficit in elderly patients. J. Am. Geriatr. Soc. 1975, 23, 433-441. [CrossRef] [PubMed]

25. NutritionQuest. Questionnaires and Screeners-Assessment \& Analysis Services-NutritionQuest. 2014. Available online: https:/ / wwwnutritionquest.com/assessment/list-of-questionnaires-and-screeners / (accessed on 30 January 2018).

26. Berdanier, C.D.; Dwyer, J.T.; Heber, D. Handbook of Nutrition and Food, 3rd ed.; CRC Press: Boca Raton, FL, USA, 2016.

27. Signorello, L.B.; Buchowski, M.S.; Cai, Q.; Munro, H.M.; Hargreaves, M.K.; Blot, W.J. Biochemical validation of food frequency questionnaire-estimated carotenoid, $\alpha$-tocopherol, and folate intakes among African Americans and non-Hispanic whites in the southern community cohort study. Am. J. Epidemiol. 2010, 171, 488-497. [CrossRef] [PubMed]

28. Guenther, P.M.; Casavale, K.O.; Reedy, J.; Kirkpatrick, S.I.; Hiza, H.A.; Kuczynski, K.J.; Kahle, L.L.; Krebs-Smith, S.M. Update of the healthy eating index: Hei-2010. J. Acad. Nutr. Diet. 2013, 113, 569-580. [CrossRef] [PubMed]

29. Bellamy, N.; Buchanan, W.W.; Goldsmith, C.H.; Campbell, J.; Stitt, L.W. Validation study of WOMAC: A health status instrument for measuring clinically important patient relevant outcomes to antirheumatic drug therapy in patients with osteoarthritis of the hip or knee. J. Rheumatol. 1988, 15, 1833-1840. [PubMed]

30. Institute of Medicine (US). Dietary Reference Intakes for Calcium and Vitamin D; The National Academies Press: Washington, DC, USA, 2011; p. 1132. 
31. Institute of Medicine (US). Dietary Reference Intakes for Vitamin A, Vitamin K, Arsenic, Boron, Chromium, Copper, Iodine, Iron, Manganese, Molybdenum, Nickel, Silicon, Vanadium, and Zinc; The National Academies Press: Washington, DC, USA, 2001; p. 800.

32. United States Department of Health and Human Services; United States Department of Agriculture. 2015-2020 Dietary Guidelines for Americans, 8th Edition, December 2015. Available online: https: / / health.gov / dietaryguidelines/2015/guidelines / (accessed on 30 January 2018).

33. United States Department of Agriculture (USDA), Center for Nutrition Policy and Promotion. The Healthy Eating Index. 1995. Available online: https://www.cnpp.usda.gov/sites/default/files/healthy_eating_ index/HEI89-90report.pdf (accessed on 30 January 2018).

34. Institute of Medicine. Dietary Reference Intakes for Energy, Carbohydrate, Fiber, Fat, Fatty Acids, Cholesterol, Protein, and Amino Acids; The National Academies Press: Washington, DC, USA, 2005; p. 1358.

35. American Heart Association, Healthy for Good, Monounsaturated Fat 2017. Available online: https:// healthyforgood.heart.org/eat-smart/articles/monounsaturated-fats (accessed on 30 January 2018).

36. American Heart Association, Healthy for Good, Polyunsaturated Fat 2017. Available online: https:// healthyforgood.heart.org/eat-smart/articles/polyunsaturated-fats (accessed on 30 July 2018).

37. Institute of Medicine. Dietary Reference Intakes for Vitamin C, Vitamin E, Selenium, and Carotenoids; The National Academies Press: Washington, DC, USA, 2000; p. 529.

38. Institute of Medicine. Dietary Reference Intakes for Thiamin, Riboflavin, Niacin, Vitamin B6, Folate, Vitamin B12, Pantothenic Acid, Biotin, and Choline; The National Academies Press: Washington, DC, USA, 1998; p. 592.

39. Tussing-Humphreys, L.M.; Fitzgibbon, M.L.; Kong, A.; Odoms-Young, A. Weight loss maintenance in African American women: A systematic review of the behavioral lifestyle intervention literature. J. Obes. 2013, 2013, 437369. [CrossRef] [PubMed]

40. Deierlein, A.L.; Morland, K.B.; Scanlin, K.; Wong, S.; Spark, A. Diet quality of urban older adults age 60 to 99 years: The cardiovascular health of seniors and built environment study. J. Acad. Nutr. Diet. 2014, 114, 279-287. [CrossRef] [PubMed]

41. United States Department of Agriculture (USDA), Center for Nutrition Policy and Promotion. Hei-2010 Total and Component Scores for Children, Adults, and Older Adults during 2011-2012. 2016. Available online: https: / / www.cnpp.usda.gov/sites/default/files/healthy_eating_index/HEI-2010-During-20112012-Oct21-2016.pdf (accessed on 30 January 2018).

42. Xu, B.; Houston, D.K.; Locher, J.L.; Ellison, K.J.; Gropper, S.; Buys, D.R.; Zizza, C.A. Higher healthy eating index-2005 scores are associated with better physical performance. J. Gerontol. Ser. A Biol. Sci. Med. Sci. 2012, 67, 93-99. [CrossRef] [PubMed]

43. Chrysohoou, C.; Panagiotakos, D.B.; Pitsavos, C.; Das, U.N.; Stefanadis, C. Adherence to the mediterranean diet attenuates inflammation and coagulation process in healthy adults: The attica study. J. Am. Coll. Cardiol. 2004, 44, 152-158. [CrossRef] [PubMed]

44. Goletzke, J.; Buyken, A.E.; Joslowski, G.; Bolzenius, K.; Remer, T.; Carstensen, M.; Egert, S.; Nothlings, U.; Rathmann, W.; Roden, M.; et al. Increased intake of carbohydrates from sources with a higher glycemic index and lower consumption of whole grains during puberty are prospectively associated with higher il-6 concentrations in younger adulthood among healthy individuals. J. Nutr. 2014, 144, 1586-1593. [CrossRef] [PubMed]

45. Ludwig, D.S. The glycemic index: Physiological mechanisms relating to obesity, diabetes, and cardiovascular disease. JAMA 2002, 287, 2414-2423. [CrossRef] [PubMed]

46. Chatzianagnostou, K.; Del Turco, S.; Pingitore, A.; Sabatino, L.; Vassalle, C. The mediterranean lifestyle as a non-pharmacological and natural antioxidant for healthy aging. Antioxidants 2015, 4, 719-736. [CrossRef] [PubMed]

47. Wang, J.; Sun, H.; Fu, Z.; Liu, M. Chondroprotective effects of alpha-lipoic acid in a rat model of osteoarthritis. Free Radic. Res. 2016, 50, 767-780. [CrossRef] [PubMed]

48. Scoditti, E.; Calabriso, N.; Massaro, M.; Pellegrino, M.; Storelli, C.; Martines, G.; De Caterina, R.; Carluccio, M.A. Mediterranean diet polyphenols reduce inflammatory angiogenesis through MMP-9 and COX-2 inhibition in human vascular endothelial cells: A potentially protective mechanism in atherosclerotic vascular disease and cancer. Arch. Biochem. Biophys. 2012, 527, 81-89. [CrossRef] [PubMed]

49. Hardin, J.A.; Cobelli, N.; Santambrogio, L. Consequences of metabolic and oxidative modifications of cartilage tissue. Nat. Rev. Rheum. 2015, 11, 521-529. [CrossRef] [PubMed] 
50. Bailey, R.K.; Fileti, C.P.; Keith, J.; Tropez-Sims, S.; Price, W.; Allison-Ottey, S.D. Lactose intolerance and health disparities among African Americans and Hispanic Americans: An updated consensus statement. J. Natl. Med. Assoc. 2013, 105, 112-127. [CrossRef]

51. Drenowatz, C.; Shook, R.P.; Hand, G.A.; Hébert, J.R.; Blair, S.N. The independent association between diet quality and body composition. Sci. Rep. 2014, 4, 4928. [CrossRef] [PubMed]

52. Fritsche, K.L. The science of fatty acids and inflammation. Adv. Nutr. 2015, 6, 293S-301S. [CrossRef] [PubMed]

53. Wang, H.J.; Zakhari, S.; Jung, M.K. Alcohol, inflammation, and gut-liver-brain interactions in tissue damage and disease development. World J. Gastroenterol. WJG 2010, 16, 1304-1313. [CrossRef] [PubMed]

54. Slavin, J.L. Dietary fiber and body weight. Nutrition 2005, 21, 411-418. [CrossRef] [PubMed]

55. Roager, H.M.; Vogt, J.K.; Kristensen, M.; Hansen, L.B.S.; Ibrügger, S.; Mærkedahl, R.B.; Bahl, M.I.; Lind, M.V.; Nielsen, R.L.; Frøkiær, H.; et al. Whole grain-rich diet reduces body weight and systemic low-grade inflammation without inducing major changes of the gut microbiome: A randomised cross-over trial. Gut 2018. [CrossRef] [PubMed]

56. Hussain, S.M.; Cicuttini, F.M.; Giles, G.G.; Graves, S.E.; Wluka, A.E.; Wang, Y. Association between dairy product consumption and incidence of total hip arthroplasty for osteoarthritis. J. Rheumatol. 2017, 44, 1066-1070. [CrossRef] [PubMed]

57. Lu, B.; Driban, J.B.; Duryea, J.; McAlindon, T.; Lapane, K.L.; Eaton, C.B. Milk consumption and progression of medial tibiofemoral knee osteoarthritis: Data from the osteoarthritis initiative. Arthritis Care Res. (Hoboken) 2014, 66, 802-809. [CrossRef] [PubMed]

58. Sahni, S.; McLean, R.R. Got osteoarthritis? Maybe milk can help. Arthritis Care Res. 2014, 66, 795-796. [CrossRef] [PubMed]

59. Janssen, H.C.; Samson, M.M.; Verhaar, H.J. Vitamin d deficiency, muscle function, and falls in elderly people. Am. J. Clin. Nutr. 2002, 75, 611-615. [CrossRef] [PubMed]

60. Houston, D.K.; Nicklas, B.J.; Ding, J.; Harris, T.B.; Tylavsky, F.A.; Newman, A.B.; Lee, J.S.; Sahyoun, N.R.; Visser, M.; Kritchevsky, S.B. Dietary protein intake is associated with lean mass change in older, community-dwelling adults: The health, aging, and body composition (Health ABC) study. Am. J. Clin. Nutr. 2008, 87, 150-155. [CrossRef] [PubMed]

61. Reedy, J.; Krebs-Smith, S.M.; Miller, P.E.; Liese, A.D.; Kahle, L.L.; Park, Y.; Subar, A.F. Higher diet quality is associated with decreased risk of all-cause, cardiovascular disease, and cancer mortality among older adults. J. Nutr. 2014, 144, 881-889. [CrossRef] [PubMed]

62. Hsiao, P.Y.; Mitchell, D.C.; Coffman, D.L.; Allman, R.M.; Locher, J.L.; Sawyer, P.; Jensen, G.L.; Hartman, T.J. Dietary patterns and diet quality among diverse older adults: The university of Alabama at Birmingham study of aging. J. Nutr. Health Aging 2013, 17, 19-25. [CrossRef] [PubMed]

63. Pieroth, R.; Rigassio Radler, D.; Guenther, P.M.; Brewster, P.J.; Marcus, A. The relationship between social support and diet quality in middle-aged and older adults in the United States. J. Acad. Nutr. Diet. 2017, 117, 1272-1278. [CrossRef] [PubMed]

64. Carlson, A.; Mancino, L.; Lino, M. Grain consumptino by Americans. In Nutrition Insight 32; USDA Center for Nutrition Policy and Promotion: Alexandria, VA, USA, 2005.

65. Juan, W.Y.L.M. Fruit and vegetable consumption by older adults. In Nutrition Insight 34; USDA Center for Nutrition Policy and Promotion: Alexandria, VA, USA, 2007.

66. Thomson, J.L.; Onufrak, S.J.; Connell, C.L.; Zoellner, J.M.; Tussing-Humphreys, L.M.; Bogle, M.L.; Yadrick, K. Food and beverage choices contributing to dietary guidelines adherence in the lower Mississippi delta. Public Health Nutr. 2011, 14, 2099-2109. [CrossRef] [PubMed]

67. Lu, B.; Driban, J.B.; Xu, C.; Lapane, K.L.; McAlindon, T.E.; Eaton, C.B. Dietary fat intake and radiographic progression of knee osteoarthritis: Data from the osteoarthritis initiative. Arthritis Care Res. (Hoboken) 2017, 69, 368-375. [CrossRef] [PubMed]

68. Champagne, C.M.; Casey, P.H.; Connell, C.L.; Stuff, J.E.; Gossett, J.M.; Harsha, D.W.; McCabe-Sellers, B.; Robbins, J.M.; Simpson, P.M.; Weber, J.L.; et al. Poverty and food intake in rural America: Diet quality is lower in food insecure adults in the Mississippi delta. J. Am. Diet. Assoc. 2007, 107, 1886-1894. [CrossRef] [PubMed]

69. Pascoe, E.A.; Smart Richman, L. Perceived discrimination and health: A meta-analytic review. Psychol. Bull. 2009, 135, 531-554. [CrossRef] [PubMed] 
70. Klesges, R.C.; Eck, L.H.; Ray, J.W. Who underreports dietary intake in a dietary recall? Evidence from the second national health and nutrition examination survey. J. Consult. Clin. Psychol. 1995, 63, 438-444. [CrossRef] [PubMed]

71. Krebs-Smith, S.M.; Graubard, B.I.; Kahle, L.L.; Subar, A.F.; Cleveland, L.E.; Ballard-Barbash, R. Low energy reporters vs others: A comparison of reported food intakes. Eur. J. Clin. Nutr. 2000, 54, 281. [CrossRef] [PubMed]

72. Alcantara, I.; Haardorfer, R.; Gazmararian, J.A.; Hartman, T.J.; Greene, B.; Kegler, M.C. Relative validation of fruit and vegetable intake and fat intake among overweight and obese African-American women. Public Health Nutr. 2015, 18, 1932-1940. [CrossRef] [PubMed]

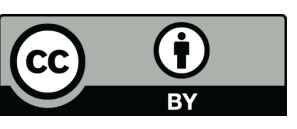

(C) 2018 by the authors. Licensee MDPI, Basel, Switzerland. This article is an open access article distributed under the terms and conditions of the Creative Commons Attribution (CC BY) license (http:/ / creativecommons.org/licenses/by/4.0/). 